\section{ofectures \\ ON SOME \\ RECENT INVESTIGATIONS \\ INTO THE \\ PATHOLOGY OF INFECTIVE AND CONTAGIOUS DISEASES.}

Delivered in connexion with the Broven Institution, at the University of London, Dec. 1879,

Br W. S. GREENFIELD, M.D. Lond., H.R.C.P., PROFESSOR SUPFRIYTENDENT OF THE BROWN INSTITUTION, ASSTSTANT-PHYSICIAN AND LECTURER ON PATHOLOGICAL ANATOMY TO ST. THOMAS'S HOSPITAL.

\section{LECTURE III._PART II}

IT will be convenient here to advert to the facts in favour of the view that the anthrax bacillus is the cause of the disease (in the ordinary sense of the word), and not a mere concomitant. These facts have not all an equal value, and are not absolute proof, but so far as they go they are strongly in favour of this view. They may be summarised as follows :-

1. The constant presence during the disease of bacilli in the blood and organs in all classes of animals. Sometimes the number of bacilli is enormous, at others small; but even in these latter cases if the fluids are used for inoculation of certain of the lower animals, the disease is sure to be attended by their development in great number. I am aware that this constant presence of bacilli has been denied by some, especially by Hiller; but I am not aware of any facts in support of Hiller's view, and it is contrary to the almost universal experience of others. Moreover, Hiller's observations refer only to the blood.

2. When blood or any animal fluid containing these bacilli or their spores, and not altered by decomposition, is inoculated, it produces the disease together with the presence of bacilli; whereas, if the fluid inoculated does not contain any bacilli or spores, it is inert. The very few assertions to the contrary do not bear the test of critical examination. My own experiments have convinced me of the accuracy of the statement here made.

3. It has been commonly asserted that, when cultivated generation after generation in indifferent media, these bacilli still retain their power of causing the disease when inoculated in susceptible animals. This I was prepared to affirm until experiment had convinced me that it was not universally true. It may be true of some cultivating media, but is not so in those I have tried. Yet the results of cultivation are such as are scarcely to be explained on the hypothesis of diluted contagium, seeing that the seventh generation may certainly produce the disease.

Another set of arguments are derived from the comparison of the effect of agents which destroy the vitality of the fungus with the causes known to prevent the disease. Any conditions, whether compression or heat, putrefaction or antiseptics, which destroy the vitality of the fungus, destroy equally the power in the fluid of producing the disease. Decomposition has an especial effect, for as it proceeds the rapidity and certainty of the effects are proportionally diminished. If, for example, two animals are inoculated with the same quantity of the same material during hot weather, the one immediately after death, the other after exposure of the material to the air for a few hours, the production of fatal symptoms is in the latter very markedly delayed. Moreover, the persistence of the contagious property for a lengthened period is coincident with, and apparently dependent upon, the formation and persistence of the bacillus spore in a condition suitable for germination.

The arguments against this view have been fully stated by Dr. Timothy Lewis, 1 and as his work not only carries weight from the position of its author, but is likely to have a wide circulation, it is desirable that I should refer in some detail

1 On the Microscopic Organisms found in the Blood of Man and Animals, and their Relation to Disease. Calcutta. 1879. No. 2967. to his views, and inquire how far they afford any ground against this view. In considering the evidence which has been adduced, showing that the virulence of septinous substance is not dependent on vegetable life, Dr. Lewis says :"The question which naturally suggests itself is, whether sufficient evidence exists to show that inoculations can be effected with like material in the absence of such living organisms. The reply to this question, so far as anthracoid and cognate diseases are concerned, is distinctly in the affirmative. ..... When Brauell published his paper in Virchow's Archiv in 1858, detailing his experiments to prove that splenic fever was an inoculable disease, he further stated the opinion that the organisms found in the blood could not be the carriers of the virus, seeing that blood not containing bacilli had been found to generate the disease. Bouley has arrived at a similar conclusion, and Böllinger, who has repeated Brauell and Bouley's experiments, has also shown that the disease may exist without the presence of bacilli in the blood, that such blood will induce the disease in other animals, and that even under such circumstances organisms may develop in the blood of the inoculated animal, and be detected during life as well as after death."

Now, let me place side by side with this Böllinger's own statement ${ }^{2}$ as to these very experiments. He says : "Brauell produced anthrax in two fowls by inoculation with blood, which did not contain any rod-like bodies, while the blood of the inoculated animal showed them. From this he drew the deduction that these structures were neither the contagious material of the disease nor the necessary carriers of the same. Bouley failed to produce anthrax by inoculating with the blood of infected animals which contained no bacteria." He goes on to say : "It is easy to conceive that bacteria in the blood and organs of diseased animals may be easily overlooked on account of their smallness," and so on. "This I have proved experimentally and with the microscope, showing that in infectious blood of this kind, without bacteria, the germs of the latter are already present in the form of spherical bacteria. Indeed, I succeeded by the inoculation of diseased blood containing bacteria in producing true anthrax, in which the blood of the diseased animals contained no bacteria, although there were bacterial germs which after death developed outside of the body into characteristic cylindrical bacteria." And in further remarks on subsequent experiments by Brauell and others he clearly shows that in speaking of the absence of bacteria he is merely referring to the rod-shaped ones, allowing that the spherical spores or germs are present. I need only quote one more sentence to show how thoroughly Böllinger accepts the view that bacteria are the cause of the disease. "The tenacity of the anthrax poison--bacteria-is exceedingly great. In the dried state bacteria are able to preserve their virulence during months, and even many years."

Bouley also is now one of the most emphatic in the assertion that bacteridia are the cause of anthrax. In a recent paper he says, "Il faut accepter aujourd'hui comne une vérité absolument démontrée que le charbon procède dela bactéridie et de la bactéridie exclusivement, puisque les expériences de laboratoire les plus rigoureuses démontrent que sans elle ou sans spores, les inoculations de matières de provenance d'animaux charbonneuses demeurent toujours stériles, tandis quelles manifestent, au contraire, leur activité, quand les bactéridies leur sont associées. D'où cette conclusion nécessaire qu'il n'y a pas de charbon possible sans bactéridies et que conséquemment, quand le charbon se manifeste quelque part, c'est que la bactéridie s'y trouve." 3

Dr. Lewis also cites against the specificity of anthrax bacillus the fact that bacilli in some respects similar may occur in the blood of healthy rats a short time after death in India, where of course the temperature is especially favourable to their development. He mentions that in the blood of rats, killed by means of chloroform or asphyxiated, he found a few hours after death short rods, which were in some cases joined end to end, and which by cultivation lengthened into longer rods, and spore formation occurred in them. $\mathrm{He}$ describes the various phases which he observed, and concludes that these bacilli are identical with those observed in anthrax. I need not weary you with a detailed description of the observations which he adduces with the object of proving this. The facts I do not for a moment dispute ; they are easy to observe for oneself ; and I have often studied and watched similar changes occurring in decomposing

2 Loc cit., p. 385

3 Receuil de Méd. Vétérinaire, Nov, 15, 1879. Paris : Asselin. B 
blood. But I think that no one who has studied cultivations of anthrax could mistake the bacilli which he figures for those cultivated from anthrax. Moreover, the whole of the arguments are pervaded by the obvious fallacy that because they are bacilli-that is, members of a genus-they are of the same species because they are found in the same fluid. That they are bacilli few could doubt; that they are anthrax bacilli, is quite another question. Anthrax bacilli do not, like these, grow in decomposing blood; moreover, these bacilli of putrefying blood, or the blood containing them, are almost entirely devoid of toxic properties, so far as my observations go ; nor has Dr. Lewis given any evidence to the contrary. I may say the same with regard to the bacilli found in urine.

Whatever value these observations may have, they cannot for a moment outweigh the results of experiments verified by a number of independent observers who have found that material from animals dying of anthrax which contains either rods or spores reproduce the disease with the utmost certainty, whilst that which does not contain either is innocuous. On the other hand, these bacilli not derived from anthrax, however elosely allied to, or even apparently identical with, the anthrax bacillus, do not when inoculated produce the disease, and in most cases are entirely innocuous.

And let me add that in order to control the results of observations on the organisms found in the blood and tissues in these diseases I have, from time to time, kept the bodies of animals which have either died a natural death or have been killed in various ways at different temperatures, and watched the organisms which developed in them.

\section{ORIGIN AND SPREAD OF ANTHRAX.}

Very naturally a large number of hypotheses have been suggested to account for the origin of the disease, or to explain its spread. Viewing it in the light of the better-known infectious diseases which affect man, we may well doubt whether it is not always carried by unsuspected ways of contagion. We cannot trace the source of every case or follow the spread of an epidemic of measles or scarlet fever; but from what we do know we feel little hesitation in affirming that the contagium has been carried from an antecedent case when we meet with an outbreak of the disease, however isolated or inexplicable that outbreak may be. In like manner, with splenic fever we are bound to exhaust all the known modes of contagion before we can be justified in believing that it is due to local conditions or processes.

Of the many ways in which contagium may be conveyed, few are so ready as that by way of food-for example, corn, hay, \&c., which are so widely distributed, and can be followed only with so much difficulty. Some few facts which have come to light support the view that the disease may be thus conveyed. Experiment has shown that the ingestion of infected material may be speedily fatal, and that this is especially so when the food contains any hard or sharp particles which can cause abrasions of the mucous membrane. Now, seeing that the anthrax bacillus can be very readily cultivated in hay infusion or in malt, we see one way in which possibly contagion might be widely distributed. But the direct evidence in favour of this mode of transmission is as yet very inconclusive.

Let me now pass to the other modes of communieation of the contagium. One very important question is the duration of the infection in the carcases of animals which have died of the disease. It is extremely difficult to understand how it comes to pass that at certain periods animals become affected in certain localities, not only without any obvious source of contagion, but often in such isolated spots and under such conditions as seem almost to negative any possibility of direct contagion. ${ }^{4}$ But since it is well known that all parts of the carcases of animals which have died from the disease are highly contagious, it is natural that the idea should have arisen that by some means the bodies of animals which have been buried in fields may infect the water, or even the pasturage, and then reproduce it. Hence the strictest regulations are in force in most conntries as to the disposal of carcases. In Prussia the carcases must be buried two metres deep ${ }^{5}$; they may not be buried in swampy places, or near running water or springs, nor in the neighbourhood of dwellings; moreover, the spot

4 Probably some of the cases of this kind which have been reported are cases of quarter-evil, a disease which has been confounded with anthrax, but which $I$ hope to show is different.

5 With quicklime one metre. must be paved or covered with stone, in order to prevent vegetation, and to keep cattle from grazing upon it. In other countries the place must be fenced in for three years, and various devices adopted for preventing any possible spread of the contagium.

I have already mentioned certain facts which point to the belief that some forms of anthrax are especially liable to occur in marshy situations, and at certain seasons of the year, when the marsh lands begin to become dried by the increasing power of the sun. All experience in fact seems to point to the great influence possessed by certain localities in favouring the occurrence and persistence of the disease. It is found that in some meadows cases occur year after year, and it has been stated that those places where bociles of anthrax victims are interred cases have arisen again and again With regard to the influence of locality, in an analogous if not identical disease, the Cape horse fever, we have very strong evidence of its marked tendency to arise amongst animals grazing in long grass on swampy ground, especially in spring and early summer. In face of these and other facts, two hypotheses might present themselves-one, that the disease originates in some miasm produced in these localities, so to speak, de novo, or developing in such a way under particular conditions as to give rise to the disease; or that the contagium is regenerated under these conditions of heat and moisture, and thus again becomes noxious. I need hardly remind you that if, as is now generally believed, the contagium consists in the bacillus anthracis, the spores of which can remain long dormant, and under favouring conditions start into life and activity we should have a state of affairs fully concordant with those observed facts of the occurrence of the disease. For we know that these bacilli would find the conditions essential to vitality in moisture, warmth, and the presence of some vegetable pabulum analogous to our cultivating fluids of the laboratory.

But it may be asked, is the pre-existence of contagium re. vivified under these conditions essential? May it not be that this bacillus can be derived from some other isomorphous bacillus, which acquires specific properties under cultivation in a particular soil or in peculiar conditions. So far as I am aware all the experiments hitherto made with the view of thus producing a contagium by cultivation of other bacilli have failed. The bacillus of hay infusion described by $\mathrm{Cohn}$ was naturally looked to as likely to be. the organism in question, but several observers have tried, without sucress, to cultivate it with this object. I do not think, however, that we ought to draw any adverse conclusion from even a very large number of failures in such experiments, for success may be possible, and if it were so a step would have been taken in the solution of the mystery of contagia far $\mathrm{ex}^{\mathrm{x}}$. ceeding any yet gained. ${ }^{6}$

But to return to the subject of infection from carcases. Gven the fact of existing spores, their persistence in the carcase or in the surrounding soil, and revivification under these favouring conditions would appear highly probable as a fresh source of disease.

Some experiments recently made by Professor Feser ${ }^{7}$ of the Munich Veterinary School have a very important bearing on this question of the duration of infection from the bodies of animals dying of splenic fever. The experiments were made especially with the view of deciding whether burial of the carcase was a sufficient precaution against the spread of the disease, or whether, as has been supposed, infection might spring up from the body after some time has elapsed. It had been suggested by Koch and others that the spores of the bacillus might be dormant and impregnate the earth around the carcase ; that, when this was in a marshy or wet ground, they would be especially liable to be spread, and become the source of reinfection. And here arose the ques tion, Whether these spores exist in the bacilliat the time of death, or were only developed afterwards? Koch suggested that, if the carcases were buried about thirty feet deep in the ground, the temperature would be too low for the development of spores, implying that the bacilli alone existed in the body at the time of death; but, as I have shown,

6 Since delivering this lecture I have, I believe, established the con verse proposition-viz., that the anthrax bacillus may be grown in cnl tivating fuids in successive generations, until it scouires an innocuons condition, though maintaining its power of germination and growth. and all its morphological characters. It may, therefore, be fairly sug sted as possible that the anthrax bacillus is only a bacillus which has acquired special properties. But this suggestion is at present purels speculative.

7 Deutsche Zeitschrift für Thiermedicin. 
spores may exist in the body, though not usually in the blood, at the time of death.

In a first series of experiments made in 1875,8 Feser found that the bodies of animals which had been buried for any length of time became innocuous. In his subsequent experiments he first of all disinterred the carcases of animals which had died of anthrax some time before, and inoculated other animals from them.

In a first set of observations he disinterred two carcases buried nearly a year before, and three others buried from four to nine days respectively, and after a very careful microscopic examination of the different parts, he inoculated other animals from them. In some cases the activity of the poison directly after death had been shown by inoculation of other animals from them, but, notwithstanding this, the experiments all proved absolutely ineffectual. It is noteworthy that Feser found in some of these carcases some bacilli which precisely resembled in their shape the bacillus anthracis.

The second series of experiments were of even more interest, as they were destined to ascertain how long after death the carcase became innocuous, and what were the parts in which the poison lingered longest.

A number of animals having died of anthrax in the Munich Veterinary School as a result of inoculation experiments which were being carried on, fifteen animals were buried in two rows one over the other, so that two could be taken up at a time, the depth being about one yard and a half. Arrangements were made for ascertaining the temperature of the soil, and the condition as to humidity. The nature of the disease and the infective power of the carcase were in every case proved by direct experiment, and in several cases the animals were inoculated in a series one from another. The record of these cases brings out some striking facts as to the variety of modes in which the poison may be introduced. Thus, in these fifteen animals, in two the disease was communicated by inoculation of spleen, which had been frozen, in one some fluid from the spleen was placed in the conjunctival sac, ${ }^{9}$ causing death in les than three days, and in one the spleen given as food produced a fatal result in fifty-six hours. Five were inoculated with the blood, either fresh or frozen, and these were much less rapidly fatal, in one case five days elapsing before death. In two cases the flesh was given as food, and rapidly caused death, and in one the flesh was used for in oculation. But the most curious result was one obtained by inoculating a sheep with some urine of another sheep which died of the disease. In the urine a large number of bodies, resembling spores of bacillus, were found. The inoculated sheep lived nine days, and then died with tetanic symptoms. The spleen was found to be greatly enlarged, measuring six inches long and one inch thick, soft and dark, and containing many spores and short characteristic anthrax rods, which, when cultivated, grew into long rods, resembling bacillus anthracis. Feser thought them identical.

One other animal was infected by inhalation ${ }^{10}$ of spores derived from the carcase of another animal, and in its carcase were found very large quantities of short anthrax bacilli, some containing spores, and also free spores.

The carcases of these animals were disinterred at periods varying from fourteen days to four months. A very elaborate microscopic examination was made of nearly all the tissues and organs, and inoculations were repeatedly made from them into other animals, but with one exception without the slightest result. The exception was in the case of the sheep buried only fourteen days. In the skin there were found spores which produced " characteristic anthrax organisms" by cultivation, but the inoculation of this material was ineffectual. Some muscle, however, rubbed down with water, was used for inoculating a horse with a fatal result in four days, with well-marked anthrax. In the exceptional instance, however, the temperature had been very low during the time of burial, and this result therefore accords with the general observation that very low temperatures are favourable to the preservation of the contagium.

There can be little doubt that these experiments go far to prove that even when under favourable conditions of warmth and moisture the carcases of animals dying of the disease do not serve as centres for the propagation of anthrax, but become inert as soon as decomposition sets in.

8 Gerlach's Archiv, Bd. iii., Hefte 5 und 6.

9 This I believe to be an unusual result, mere application to the con-

junctiva rarely producing the disease.
Another set of observations seem also to show that, as had already been shown by others, vegetables of various kinds grown orer the carcases are perfectly harmless. Nor did inoculation with the soil around the body produce any result. It may therefore be safely assumed that some other mode of origin must be sought.

Notwithstanding the negative results of these experiments, it may yet be an open question whether in certain cases the contagium does not persist in the surrounding soil. The ex periments of MI. Pasteur just published, though far less extensive than those of Feser, can hardly be entirely set aside.

In these experiments MI. Pasteur, with MIM. Chamberlaud ${ }^{11}$ and Roux, succeeded in reproducing anthrax by means of earth from the soil and around the carcase of an animal which had died of anthrax some months before, and also, as he states, cultivating anthrax bacillus in earth and infecting other earth from that over the carcase.

He first cultivated in 500 grammes of earth free from germs of bacteridia, 20 drops of anthrax blood, and after thoroughly mixing the earth, he took 2 grammes of it and added it to 300 grammes of fresh earth; then from this he took $\mathbf{5}$ grammes and mixed it with 100 grammes of fresh earth, also free from bacteridia. From the latter he took five grammes and was able to extract from it "germs of bacteridia," of which he proved the virulence by inoculation of guinea-pigs. The time from the commencement of the experiments to the inoculation were from June to September. These earth cultivations, I may point out, only reached what one may call the second generation of earth cultivation.

The second series of experiments were made by burying the carcase of a sheep which had died of splenic fever, and ten months after (in May, 1879) he took earth from the superficial and deep layers of earth from the place of burial, and by inoculation of guinea-pigs with the superficial layer he produced charbon, whilst those from the deep layers caused septicæmia, which was, he says, of peculiar character.

These experiments prove conclusively, according to $\mathbf{M}$. Pasteur, that the soil may be the means of preservation and cultivation of the germs and spores of bacteria, and that from them after some months the disease may be reproduced. The endemic prevalence of anthrax is thus, in his opinion, fully accounted for, the earth being infected by numerous cases. I must confess that I myself believe that, in spite of the elaborate precautions sometimes enforced to prevent the persistence and spread of the disease, there is sufficient evidence that the careless disposal of portions of the carcasee.g., of parts of the skin and hair-is very common, and that this affords a sufficient explanation of the persistence and virulence of the disease.

One or two other points in relation to this disease remain to be mentioned, one the possibility of inoculating anthrax without causing a fatal result, and the protection conferred by this attack; the other the relation of the disease as it occurs in the lower animals to human disease.

In some experiments made last year by Dr. Sanderson and Mr. Duguid, but not yet published, ${ }^{12}$ they found that when anthrax material, which had been transmitted through rodents, especially the guinea-pig, was inoculated in cows, the symptoms produced were analogous to those of anthrax, and very intense, but that in no case was the disease fatal This was equally the case, whether the material was derived directly from the guinea-pig, or by means of cultivation in aqueous humour or hay infusion.

This observation seemed to me a very important one, for this reason, that if anthrax is, as is supposed by some, an acute specific fever, such inoculation of a modified contagium might be found to confer protection from subsequent attack. And, collaterally, if it did so confer protection, the marked analogy thus established with acute specific fevers would point to the possibility of the contagium of other acute specifies being of bacterial nature.

I have, therefore, repeated the experiment, as yet only with the inoculation through guinea-pigs, for I have not yet had any opportunity of further investigation with original material. The experiment was as follows. A heifer, previously in good health, having been kept under observation for nearly three weeks to ascertain its normal temperature, was inoculated with a small quantity of anthrax virus, which from some cause was ineffectual. The animal was therefore reinoculated with a larger quantity of material, the second cultivation in hay infusion, containing abundant spores, the

11 Bull. de l'Acad. de Médecine, Dec. 1879

12 They have since been publiske 1 in the Journal of the Royal Agricultural Society, vol. i., 1880. 
activity of which was at the same time tested by inoculation on a mouse. The course of the symptoms is best indicated by the chart which I have placed before you. For the first twenty-four hours no symptoms were observed; there was only very slight swelling at the seat of inoculation. On the morning of the second day--i.e., about forty hours after the inoculation-the rectal temperature was found to be $104^{\circ}$, having previously been $100^{\circ}$ to $101^{\circ}$ (the normal temperature). The animal was drowsy and stupid, and fed badly, but no rigor was observed, nor any very special symptoms. In the evening of the same day the temperature was lower $\left(1024^{\circ}\right)$, but on the following day it rose to $1064^{\circ} \mathrm{F}$., and continued to vary between $105^{\circ}$ and $107^{\circ}$ for three days, during which time the animal was seriously ill, and at times appeared hardly likely to survive. On the eighth day, however, the temperature fell to $104^{\circ}$, and on the ninth to $101^{\circ}$, after which the animal speedily recovered, and continued perfectly well.

I was unable to discover any bacilli in the blood, but I do not attach any importance to this fact in proving that they did not exist. Inoculation with this blood, not containing bacilli, did not produce any symptoms in a rabbit. ${ }^{13}$

It remains to be seen whether this modified attack will confer any future protection on the animal.

ANTHRAX IN RELATION TO HUMAN DISEASE.

We know that in districts where anthrax is prevalent amongst cattle cases of malignant carbuncle appear in the human subject from time to time, especially in those engaged in dressing carcases, or who have to do with the skins of the animals. I must not enter into the forms which this disease assumes in man, which have been very fully described by Böllinger, ${ }^{1.4}$ though I believe that several different diseases are included by him under the name of anthrax.

But I wish to point out the very great probability that some of the anomalous forms of pyæmia in the human subject, such as what is called angina Ludovici, for which no cause is discoverable during life or after death, may be due to contagion from this disease. One fact alone may serve as an illustration of the great facilities which exist for such contagion. In a case of anthrax, from which the material for some of my experiments was derived, the carcase had already been dressed for the London market. Some of the blood which had drained from it on to the floor was taken, and a guinea-pig inoculated with a very minute quantity. The animal died in twenty-seven hours, and its blood swarmed with bacilli. It was well known that the animal had died of splenic fever. I need hardly emphasise the urgent need of more thorough sanitary inspection. Moreover, there is some evidence that there exist forms of blood-poisoning in connexion with certain tredes in this country which are really derived from anthrax, such as probably the "woolsorter's disease," of which Dr. Bell of Bradford has just reported a case in which he found anthrax bacilli in the blood ${ }^{15}$ (The LANCET, Dec. 27th, 1879, p. 579). Similar diseases are known to occur in like occupations on the Continent. But these forms are as yet very little known; the two diseases in which there is a local lesion-viz., malignant pustule and so-called "internal anthrax"-being the best known in relation to human diseases.

I must now pass to the other anthracoid diseases which affect the lower animals.

13 Since the delivery of this lecture these experiments have been continued, and some of them have been detailed in the Journal of the Royal Agricultural Society (Part I. 1880). These, together with still further experiments not yet published, show that protection is conferred, which is great in degree and lasts a considerable time; and that this is true whether the poison is directly drawn from the guinea-pig or is cultivated, provided only that symptoms are produced by the first inoculation.

14 Ziemssen's Encyclopædia, vol. iii.

15 I may mention that $\mathrm{I}$ have been able to prove the identity of this disease with the splenic fever of cattle by recent experiments.

Coombe Lying-In Hospital, Dublin.-The annual meeting of this institution was held recently, and from the report we learn that during the past year 680 patients were under treatment in the hospital, and 1953 attended in their confinements at their own homes. There were also 1754 attendances at the special dispensary for diseases peculiar to women, and 5448 at the general dispensary. Of the 680 intern patients 559 were in the labour wards, and 121 in the wards for diseases of women, the larger number of the latter having to undergo operations of a serious character. Since the enlargement of the hospital an increased expenditure has arisen, and a debt of some $£ 700$ has been incurred, which the Board hope shortly to discharge.
ON THE PATHOGENY OF LEAD PALSY.

By A. DE WATTEVILLE, M.A., B.Sc, M.R.C.S. ENG. ASSISTANT PHYSICLN TO THE MOSPITAL FOR EPILTSSY AND PARALTSIS, \&C.

ONE of the most characteristic symptoms of saturnism, or lead-poisoning, is the paralysis of certain muscles of the forearm giving rise to the condition known as wrist-drop, or dropped hand. This condition is readily recognised by the fact that the patient cannot extend the hand upon the arm nor the first phalanges upon the metacarpal bones. The hand held out in the prone position hangs down helplessly the only movements possible are a weak flexion and extension of the distal phalanges of the fingers and thumb. At first sight we have the spectacle offered by a paralysis of the musculo-spiral nerve from injury at the point of its emergence from between the fibres of the triceps; and the question naturally arises, To which of the possible causes is the paralysis due? If the patient presents the patho gnomonic blue line along the gums-the peculiar aspect of saturnine anæmia; if he complains of colic and constipa. tion, or if he is known to have been much exposed to the deleterious influence of the poison, then we naturally conclude that he suffers from lead palsy. But we must not forget, on the one hand, the possible concomitance of lead symptoms with a simple paralysis of the musculo-spiral from exposure to cold or slight pressure ; nor, on the other hand, the possibility of a lead palsy unaccompanied by any of the other symptoms first mentioned. The patient may have been unconsciously poisoned by contaminated water, snuff, and the like, or by adulterated hair-dyes, toilet-powder, and other cosmetics, or even by medicinal use of acetate of lead, as I once observed in a case; and the first warning of the intoxication may be the paretical condition of the forearm. Now, in order to clear up our doubts, we are generally told to have recourse to two tests-that of the electrical reactions of the affected nerve and muscles, and that of the nonimplication of the supinator longus. A discussion of the first will be best reserved for another opportunity; my object here is to consider the second, with a view of elucidating a point hitherto somewhat neglected in our text-books ; I mean the pathogeny and localisation of lead paralysis. To Duchenne belongs the credit of having stated as a general axiom that in lead paralysis of the usual type the supinators always escape, though all the other muscles supplied by the musculo-spiral nerve are liable to be affected. The supinator longus, as he showed, is mainly a flexor of the forearm ; and the method of ascertaining its condition is to tell the patient to flex the arm, whilst the observer resists the motion by holding the limb in a position midway between pronation and supination. Under these conditions the belly of the muscle, if unimpaired, starts prominently for ward, and is readily seen and felt as a hard triangular mass filling up the bend of the elbow. We shall see, however, that this non implication of the supinators among the muscles supplied by the musculo-spiral can no longer be held as a pathognomonic feature of lead paralysis; for it may also be observed in certain cases of circumscribed an. terior cervical poliomyelitis - that is, of inflammation or sclerosis of certain portions of the anterior horn of grey matter in the cervical enlargement-and more especially of certain groups of the large multipolar or ganglionic cells therein contained.

I $t$ is certainly strange that such a phenomenon, the uni= form protection of a muscle amidst the general paralysis and atrophy of all its companions, supplied by the very same nerve, should have been so long recognised without eliciting any attempts at an explanation. Yet this is what has happened ; and it is only lately that $\mathbf{E}$. Remak, in a valuable paper has propounded what appears to me to be a most satisfactory hypothesis. Most movements, such as flexion or extension of the several segments of a limb upon one another, although apparently very simple, require the cooperation of several muscles. The centres for these primitive associations are in the anterior grey matter of the cord, which contains the large cells just mentioned. These cells, arranged in groups, more or less independent physiologically and pathologically, are connected with the brain through 